\title{
New regular black hole solutions
}

\author{
José P. S. Lemos* and Vilson T. Zanchin ${ }^{\dagger}$ \\ ${ }^{*}$ Centro Multidisciplinar de Astrofísica - CENTRA, Departamento de Física, Instituto Superior \\ Técnico - IST, Universidade Técnica de Lisboa - UTL, Av. Rovisco Pais 1, 1049-001 Lisboa, \\ Portugal, \& \\ Institute of Theoretical Physics - ITP, Freie Universität Berlin, Arnimallee 14 D-14195 Berlin, \\ Germany. \\ ${ }^{\dagger}$ Centro de Ciências Naturais e Humanas, Universidade Federal do ABC, Rua Santa Adélia, 166, \\ 09210-170, Santo André, SP, Brazil
}

\begin{abstract}
In the present work we consider general relativity coupled to Maxwell's electromagnetism and charged matter. Under the assumption of spherical symmetry, there is a particular class of solutions that correspond to regular charged black holes whose interior region is de Sitter, the exterior region is Reissner-Nordström and there is a charged thin-layer in-between the two. The main physical and geometrical properties of such charged regular black holes are analyzed.
\end{abstract}

Keywords: Charged black holes, Regular black holes, Einstein-Maxwell

PACS: $4.70 \mathrm{Bw}, 04.20 . \mathrm{Jb}, 04.40 \mathrm{Nr}$

\section{INTRODUCTION}

An early motivation to find regular black hole models was to get rid of spacetime singularities. Such black holes would substitute the usual Schwarzschild and ReissnerNordström black holes with singularities in their center. Gliner in 1966 [1] proposed that singularities can be avoided by matter with an inflationary equation of state, with a de Sitter core, and that a spacetime filled with a false vacuum inside a horizon could provide a description of the final state of gravitational collapse. Bardeen, in 1968 [2] put forward a solution of Einstein's equations in which there was a black hole with horizons and without a singularity. The matter is magnetically charged, and it yields a modification of the Reissner-Nordström metric. Near the center it turns, in a natural smooth way, into a de Sitter core. Gliner's ideas were further developed by himself and Dymnikova [3], while Bardeen's solutions were further explored by Ayón-Beato and García [4]. There were some tries to join directly a de Sitter vacuum onto the Schwarzschild solution, but proved to be incorrect [5, 6] (see also [7] for further incorrect matchings). Further developments were performed in [8]. Borde understood the topology and causality of these regular solutions [9]. For a review see [10].

In this work we find a new regular black hole. It is a regular charged black hole in which the interior de Sitter metric is matched to the exterior Reissner-Nordström metric. The electric charge is in an energyless coat located at the boundary of the de Sitter core. The paper is organized as follows. In Sec. 2 we display the basic equations of the system. In Sec. 3 we build the regular charged black hole, and in Sec. 4 we analyze the main properties of the solution. 


\section{BASIC EQUATIONS}

The cold charged fluids considered in the present work are described by EinsteinMaxwell equations with matter,

$$
\begin{aligned}
& G_{\mu v}=8 \pi\left(T_{\mu v}+E_{\mu v}\right), \\
& \nabla_{v} F^{\mu v}=4 \pi J^{\mu},
\end{aligned}
$$

where Greek indices $\mu, v$, etc., run from 0 to 3. $G_{\mu v}=R_{\mu v}-\frac{1}{2} g_{\mu v} R$ is the Einstein tensor, with $R_{\mu \nu}$ being the Ricci tensor, $g_{\mu \nu}$ the metric, and $R$ the Ricci scalar. We have put both the speed of light $c$ and the gravitational constant $G$ equal to unity throughout. $E_{\mu \nu}$ is the electromagnetic energy-momentum tensor, which can be written in the form $4 \pi E_{\mu \nu}=F_{\mu} \rho^{\rho} F_{\nu \rho}-\frac{1}{4} g_{\mu \nu} F_{\rho \sigma} F^{\rho \sigma}$, where the Maxwell tensor is $F_{\mu v}=\nabla_{\mu} \mathscr{A}_{v}-\nabla_{\nu} \mathscr{A}_{\mu}$, $\nabla_{\mu}$ representing the covariant derivative, and $\mathscr{A}_{\mu}$ the electromagnetic gauge field. In addition, $J_{\mu}=\rho_{\mathrm{e}} U_{\mu}$, is the current density, $\rho_{\mathrm{e}}$ is the electric charge density, and $U_{\mu}$ is the fluid velocity. $T_{\mu \nu}$ is the material energy-momentum tensor, which, for the purpose of the present work, is taken in the form of an isotropic fluid $T_{\mu \nu}=\left(\rho_{\mathrm{m}}+p\right) U_{\mu} U_{v}+p g_{\mu \nu}$, where $\rho_{\mathrm{m}}$ is the fluid matter-energy density, $p$ is the isotropic fluid pressure. We are interested in a false vacuum state where the energy-momentum tensor satisfies the relations $T_{0}^{0}+E_{0}^{0}=T_{1}^{1}+E_{1}^{1}$, and $T_{2}^{2}+E_{2}^{2}=T_{3}^{3}+E_{3}^{3}$.

We assume that the spacetime is static and spherically symmetric, so that the metric is conveniently written in the Schwarzschild-like form, namely,

$$
d s^{2}=-B(r) d t^{2}+A(r) d r^{2}+r^{2} d \Omega^{2} .
$$

where $r$ is the usual radial coordinate, $A$ and $B$ are function of $r$ only, and $d \Omega^{2}$ is the metric of the unit sphere. The gauge field $\mathscr{A}_{\mu}$ and the four-velocity $U_{\mu}$ have the form

$$
\mathscr{A}_{\mu}=-\phi(r) \delta_{\mu}^{t}, \quad U_{\mu}=-\sqrt{B(r)} \delta_{\mu}^{t},
$$

where $\phi(r)$ is the electric potential.

The relevant Einstein-Maxwell field equations for the metric (3) can now be found. The only nonzero component of Maxwell equations (2) furnishes

$$
Q(r)=4 \pi \int_{0}^{r} \rho_{\mathrm{e}}(r) \sqrt{A(r)} r^{2} d r=\frac{r^{2} \phi^{\prime}(r)}{\sqrt{B(r) A(r)}},
$$

where an integration constant was set to zero. $Q(r)$ is the total electric charge inside the surface of radius $r$.

The $t t$ and $r r$ components of Einstein equations (1) furnish the following relations

$$
\begin{aligned}
& \frac{B^{\prime}(r)}{B(r)}+\frac{A^{\prime}(r)}{A(r)}=8 \pi r A(r)\left[\rho_{\mathrm{m}}(r)+p(r)\right], \\
& \left(r A^{-1}(r)\right)^{\prime}=1-8 \pi r^{2}\left(\rho_{\mathrm{m}}(r)+\frac{Q^{2}(r)}{8 \pi r^{4}}\right),
\end{aligned}
$$


where the prime denotes the derivative with respect to the coordinate $r$. The conservation equations $\nabla_{v} T^{\mu \nu}=0$, together with Maxwell equations, give

$$
2 p^{\prime}(r)+\frac{B^{\prime}(r)}{B(r)}\left[\rho_{\mathrm{m}}(r)+p(r)\right]-2 \frac{\phi^{\prime}(r) \rho_{\mathrm{e}}(r)}{\sqrt{B(r)}}=0,
$$

which is the only non-identically zero component of the conservation equations. The other nonzero components of Einstein-Maxwell equations give an additional relation which is not independent of the above set of equations.

\section{REGULAR CHARGED BLACK HOLES}

Consider that there is matter up to the boundary radius $r_{0}$. We now do the following hypothesis

$$
\rho_{\mathrm{m}}(r)+p(r)=0
$$

valid for $r \leq r_{0}$, and for $r \geq r_{0}$, since vacuum always satisfies (9). With such a condition, the metric coefficients result in $B(r)=A^{-1}(r)$. Moreover, after the assumption (9), Eqs. (8) and (5) give

$$
p^{\prime}(r)=\frac{\phi^{\prime}(r) \rho_{\mathrm{e}}(r)}{\sqrt{B(r)}}=\frac{Q Q^{\prime}}{4 \pi r^{4}} .
$$

This means that $p^{\prime}(r)$ is proportional to the charge density $\rho_{e}(r)$ and may be interpreted as the charge density giving rise to a pressure gradient.

To complete the set up we need another input. One of the simplest ansatz one can make is through the following equation

$$
8 \pi \rho_{\mathrm{m}}(r)+\frac{Q^{2}(r)}{r^{4}}=\frac{3}{R^{2}}
$$

for $r \leq r_{0}$, and where $R$ is a constant to be determined. This resembles the interior Schwarzschild assumption of constant energy density.

In order for displaying the solution in a simple form, let us define, the $\theta$ function as usual by $\theta\left(r-r_{0}\right)=1$ when $r-r_{0} \geq 0$ and $\theta\left(r-r_{0}\right)=0$ when $r-r_{0}<0$. Then we can write all of the functions in succinct form. The metric is now

$$
d s^{2}=-B(r) d t^{2}+B^{-1}(r) d r^{2}+r^{2} d \Omega^{2},
$$

where

$$
\begin{gathered}
B(r)=B_{\mathrm{i}} \theta\left(r_{0}-r\right)+B_{\mathrm{e}}\left[1-\theta\left(r_{0}-r\right)\right], \\
B_{\mathrm{i}}=\left(1-\frac{r^{2}}{R^{2}}\right), \quad B_{\mathrm{e}}=\left(1-\frac{2 m}{r}+\frac{q^{2}}{r^{2}}\right) .
\end{gathered}
$$


$R$ thus defines a de Sitter radius. The electric potential is given by

$$
\phi(r)=-\frac{q}{r_{0}} \theta\left(r_{0}-r\right)-\frac{q}{r}\left[1-\theta\left(r_{0}-r\right)\right] .
$$

The other important quantities can be written as follows

$$
\begin{aligned}
& Q(r)=q \theta\left(r-r_{0}\right), \quad Q^{\prime}(r)=q \delta\left(r-r_{0}\right), \quad \rho_{\mathrm{e}}(r)=\frac{q \sqrt{B(r)}}{4 \pi r^{2}} \delta\left(r-r_{0}\right), \\
& 8 \pi \rho_{\mathrm{m}}(r)=\left(\frac{3}{R^{2}}-\frac{Q^{2}(r)}{r^{4}}\right) \theta\left(r_{0}-r\right), \\
& 8 \pi p(r)=\left(-\frac{3}{R^{2}}+\frac{Q^{2}(r)}{r^{4}}\right) \theta\left(r_{0}-r\right), \quad p^{\prime}(r)=\frac{q^{2}}{4 \pi r^{4}} \delta\left(r-r_{0}\right) .
\end{aligned}
$$

This solution represents regular black holes with a de Sitter core, an electric energyless matter coat at $r_{0}$, and Reissner-Nordström all the way up.

The solution has four parameters: $m, q, r_{0}$ and $R$. There are a few relations among them. These relations follow from imposing appropriate junction conditions at the boundary $r=r_{0}$. Indeed one can show that the boundary at $r_{0}$ can be really a boundary surface. For that, the metric $g_{\mu \nu}$ (or the first fundamental form), and the extrinsic curvature (or the second fundamental form) $K_{\mu v}$, should be continuous at the surface, i.e., $g_{\mu \nu}^{+}=g_{\mu \nu}^{-}$, and the extrinsic $K_{\mu \nu}^{+}=K_{\mu \nu}^{-}$, where \pm represent the exterior and interior regions. The matching of the first fundamental form requires that in Eq. (13) $B_{\mathrm{i}}=B_{\mathrm{e}}$, i.e., $1-\frac{r_{0}^{2}}{R^{2}}=1-\frac{2 m}{r_{0}}+\frac{q^{2}}{r_{0}^{2}}$. This implies in the first relation among $m, q, r_{0}$, and $R, \frac{1}{R^{2}}=\frac{1}{r_{0}^{3}}\left(2 m-\frac{q^{2}}{r_{0}}\right)$. The matching of $K_{\mu \nu}$ implies in the continuity of the first derivative of $B_{\mathrm{i}}(r)$ and $B_{\mathrm{e}}(r)$ at $r=r_{0}$, that is, $\frac{r_{0}}{R^{2}}=\frac{m}{r_{0}^{2}}-\frac{q^{2}}{r_{0}^{3}}$. This, together with the first fundamental form equation, gives two other simplified equations (assume positive $q$ )

$$
R=\sqrt{3} r_{0}^{2} \frac{1}{q}, \quad m=\frac{2}{3 r_{0}} q^{2} .
$$

So there are only two free parameters, $r_{0}$ and $q$ say. From Eq. (19) we see that as $r_{0}$ increases the mass $m$ decreases. This is due to the fact that the pressure, and thus the density, decrease fast as $r_{0}$ increases. When there is no charge there is no black hole since $q=0$ implies $m=0$, leaving a Minkowski spacetime alone.

From Eqs. (13) and (14) one can infer that

$$
r_{0} \leq R
$$

otherwise the matter boundary would be outside its de Sitter horizon. One can also infer from $\frac{1}{R^{2}}=\frac{1}{r_{0}^{3}}\left(2 m-\frac{q^{2}}{r_{0}}\right)$ that, when $r_{0} \leq R$, then $1-\frac{2 m}{r_{0}}+\frac{q^{2}}{r_{0}^{2}} \geq 0$ which means that if there are solutions these solutions are such that, either $r_{0}$ is inside or on the inner (or Cauchy) horizon $r_{-}$, or $r_{0}$ is outside or on the outer (event) horizon $r_{+}$. Since we want black holes we should search for solutions in which $r_{0} \leq r_{-}$, but the procedure dictates what kind of solutions there are. 


\section{THE PROPERTIES OF THE SOLUTIONS}

Since we are not interested in $q=0$ we can parametrize all quantities in terms of $q$. From Eqs. (19)-(20) one finds

$$
r_{0} \geq \frac{\sqrt{3}}{3} q
$$

Now, we have to compare $r_{0}$ with $r_{-}$and $r_{+}$. In the present case, these are given by

$$
r_{ \pm}=m \pm \sqrt{m^{2}-q^{2}}=\left(\frac{2 q}{3 r_{0}} \pm \sqrt{\frac{4 q^{2}}{9 r_{0}^{2}}-1}\right) q .
$$

These radii, together with the line for $r_{0} / q$, are shown in Fig. 1. The solutions can then be divided into four classes (for details see [11]):

1. Regular nonextremal black holes with a null boundary: When $r_{0} / q$, i.e., $r_{0}$ (since we are seeing it for fixed charge) has its minimum value $r_{0} / q=\sqrt{3} / 3$, then the radius of the matter coincides with the Cauchy horizon $r_{-}$. There is matter up to the null surface $r_{-}$and then two horizons [6]. A perfectly regular black hole.

2. Regular nonextremal black holes with a timelike boundary: For larger $r_{0} / q$, i.e., larger $r_{0}$, the Cauchy horizon $r_{-}$grows faster and remains outside the radius of the matter, $r_{0}<r_{-}$. There is matter up to $r_{0}$ and then outside the matter stand two horizons. Also a perfectly regular black hole.

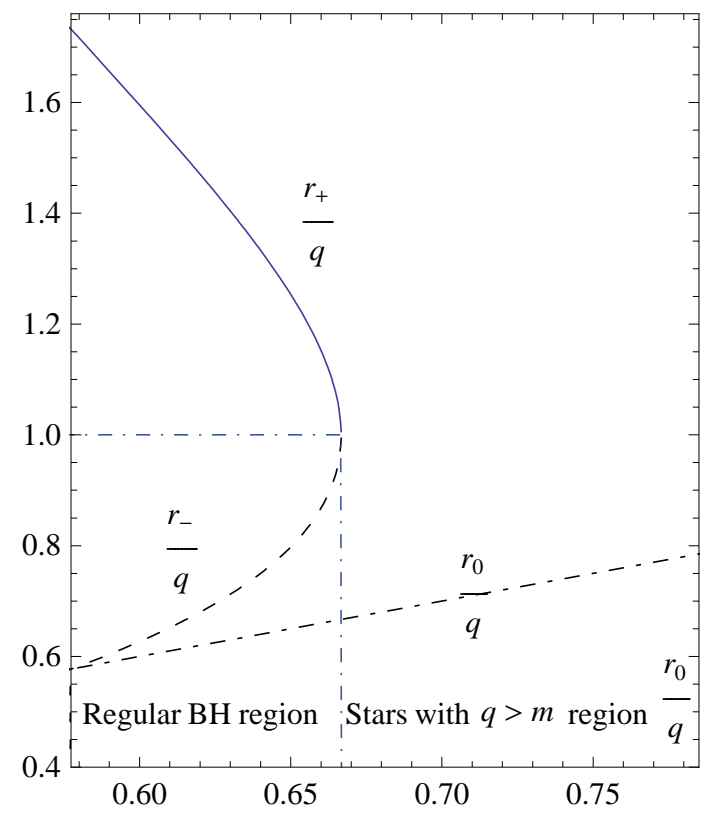

FIGURE 1. A plot of several radii as a function of $r_{0} / q$. The minimum value of $r_{0} / q$ is $\sqrt{3} / 3$, and the vertical dashed-dotted line is at $r_{0} / q=2 / 3$.

3. Regular extremal black holes with a timelike boundary: When $r_{0} / q=2 / 3$ then the Cauchy and event horizons coincide, it is an extremal regular black hole. The horizon is now at the furthest coordinate distance from the surface of the matter at $r_{0}$. This means that $r_{0}$ cannot be the radius of the horizon of an extremely charged Reissner-Nordström 
black hole (see Fig. 1). In fact, as found in Ref. [12], in the extremely charged limit in which the Cauchy and the event horizons are identical, $r_{-}=r_{+}$, if the boundary of the de Sitter region is pushed to the double horizon, i.e., in the limit $r_{0} \rightarrow r_{+}=r_{-}$, the resulting solution is a quasiblack hole, and not a regular black hole.

4. Regular overcharged stars with a timelike boundary: For $r_{0} / q>2 / 3$ there are no black holes. An overcharged star (i.e. charge greater than mass) pops up with no horizons. They have disappeared. It is funny that $r_{0}$ of the first star is still less than $r_{-}$of the extremal regular black hole. Perhaps this is no surprise as we are familiar with the fact that the Reissner-Nordström solution has the same feature, after the extremal horizon the singularity at $r=0$ becomes bare.

For a range of parameters, the solution is thus a regular electrically charged black hole. It is built from false vacuum up to, but not at, $r_{0}$, at $r_{0}$ there is a thin electrical layer of an energyless field, and outside is pure Reissner-Nordström, with two horizons at $r_{-}$and $r_{+}$. The radius to charge ratio and mass to charge ratio of the solution is well defined. The metric for $r<r_{0}$ is the de Sitter metric, where the isotropic pressure is constant $\left(p(r)=-\rho_{\mathrm{m}}(r)=3 / 8 \pi R^{2}\right)$ in the region inside the surface $r=r_{0}$, and goes to zero at $r_{0}$. Furthermore, since the charge density $\rho_{\mathrm{e}}(r)$ is a Dirac delta function centered in $r=r_{0}$, the total charge $q$ is distributed uniformly on the surface $r=r_{0}$. The limit of zero charge is not a regular black hole, it is Minskowski spacetime.

\section{ACKNOWLEDGMENTS}

This work was partially funded by Fundação para a Ciência e Tecnologia (FCT) Portugal, through projects No. CERN/FP/109276/2009 and PTDC/FIS/098962/2008. JPSL thanks the FCT grant SFRH/BSAB/987/2010. VTZ thanks Fundação de Amparo à Pesquisa do Estado de São Paulo (FAPESP) and Conselho Nacional de Desenvolvimento Científico e Tecnológico of Brazil (CNPq) for financial help.

\section{REFERENCES}

1. E. Gliner, Sov. Phys. JETP 22, 378 (1966).

2. J. Bardeen, in Proceedings of GR5, Tbilisi, URSS, 1968.

3. I. G. Dymnikova, Gen. Relativ. Gravit. 24, 235 (1992).

4. E. Ayón-Beato and A. García, Phys. Rev. Lett. 80, 5056 (1998).

5. O. Gron, Lett. Nuovo Cim. 44 (1985) 177.

6. E. Poisson and W. Israel, Phys. Rev. D 41, 1796 (1990).

7. D. V. Gal'tsov and J. P. S. Lemos, Class. Quant. Grav. 18, 1715 (2001).

8. K. A. Bronnikov, H. Dehnen, and V. N. Melnikov, Gen. Rel. Grav. 39, 973 (2007).

9. A. Borde, Phys. Rev. D 55, 7615 (1997).

10. S. Ansoldi, arXiv:0802.0330 [gr-qc] (2008).

11. J. P. S. Lemos and V. T. Zanchin, "Regular black holes: electrically charged solutions, ReissnerNordström outside the core", to be published (2011).

12. J. P. S. Lemos and V. T. Zanchin, Phys. Rev. D 81, 124016 (2010). 
Copyright of AIP Conference Proceedings is the property of American Institute of Physics and its content may not be copied or emailed to multiple sites or posted to a listserv without the copyright holder's express written permission. However, users may print, download, or email articles for individual use. 